Research article

\title{
Variants in estrogen-biosynthesis genes CYP17 and CYP19 and breast cancer risk: a family-based genetic association study
}

\author{
Habibul Ahsan ${ }^{1,2}$, Alice S Whittemore ${ }^{3}$, Yu Chen ${ }^{1}$, Ruby T Senie ${ }^{1,2}$, Steven P Hamilton ${ }^{4}$, \\ Qiao Wang ${ }^{2}$, Irina Gurvich ${ }^{2}$ and Regina M Santella2,5
}

\author{
1'Department of Epidemiology, Mailman School of Public Health, Columbia University, New York, New York, USA \\ ${ }^{2}$ Herbert Irving Comprehensive Cancer Center, Columbia University, New York, New York, USA \\ 3Division of Epidemiology, Department of Health Research and Policy, Stanford University School of Medicine, Stanford, California, USA \\ 4Department of Psychiatry, Columbia University College of Physicians and Surgeons and the New York State Psychiatric Institute, New York, New \\ York USA \\ ${ }^{5}$ Department of Environmental Health Sciences, Mailman School of Public Health, Columbia University, New York, New York, USA
}

Corresponding author: Habibul Ahsan, ha37@columbia.edu

Received: 10 Jun 2004 Revisions requested: 2 Aug 2004 Revisions received: 2 Sep 2004 Accepted: 29 Sep 2004 Published: 11 Nov 2004

Breast Cancer Res 2005, 7:R71-R81 (DOI 10.1186/bcr951)

(C) 2004 Ahsan et al. licensee BioMed Central Ltd.

This is an Open Access article distributed under the terms of the Creative Commons Attribution License (http://creativecommons.org/licenses/by/ 2.0), which permits unrestricted use, distribution, and reproduction in any medium, provided the original work is properly cited.

\begin{abstract}
Background Case-control studies have reported inconsistent results concerning breast cancer risk and polymorphisms in genes that control endogenous estrogen biosynthesis. We report findings from the first family-based association study examining associations between female breast cancer risk and polymorphisms in two key estrogen-biosynthesis genes CYP17 ( $\rightarrow \mathrm{C}$ promoter polymorphism) and CYP19 (TTTA repeat polymorphism).

Methods We conducted the study among 278 nuclear families containing one or more daughters with breast cancer, with a total of 1123 family members (702 with available constitutional DNA and questionnaire data and 421 without them). These nuclear families were selected from breast cancer families participating in the Metropolitan New York Registry, one of the six centers of the National Cancer Institute's Breast Cancer Family Registry. We used likelihood-based statistical methods to examine allelic associations.
\end{abstract}

Results We found the CYP19 allele with 11 TTTA repeats to be associated with breast cancer risk in these families. We also found that maternal (but not paternal) carrier status of CYP19 alleles with 11 repeats tended to be associated with breast cancer risk in daughters (independently of the daughters' own genotype), suggesting a possible in utero effect of CYP19. We found no association of a woman's breast cancer risk either with her own or with her mother's CYP17 genotype.

Conclusion This family-based study indicates that a woman's personal and maternal carrier status of CYP19 11 TTTA repeat allele might be related to increased breast cancer risk. However, because this is the first study to report an association between CYP19 11 TTTA repeat allele and breast cancer, and because multiple comparisons have been made, the associations should be interpreted with caution and need confirmation in future family-based studies.

Keywords: breast cancer, cyp17, cyp19, estrogen biosynthesis genes, family-based design

\section{Introduction}

Cumulative exposure to circulating estrogen is considered to be of primary importance in breast cancer etiology. Estrogen biosynthesis, cellular binding and metabolism involve many steps, and the genes controlling these steps may contribute to inherent variability in breast cancer susceptibility. Endogenous estrogen is produced predominantly in the ovarian theca cells in premenopausal women and in the breast stromal adipose cells in postmenopausal women. The present study focuses on CYP17 and CYP19, two key genes that control the biosynthesis of estradiol and estrones from their lipid precursors and are expressed in these cells. CYP1 7 controls two successive early steps of endogenous estrogen biosynthesis by converting pregnenolone and progesterone to precursors of androgen and estrogen. CYP19, also known as aromatase, controls the terminal step of estrogen biosynthesis by converting 19- 
carbon steroids (testosterone and androstenedione) to 18carbon estrogens (estradiol and estrone).

A $T \rightarrow C$ single-nucleotide polymorphism in the 5 ' promoter region of the CYP17 gene and a TTTA repeat polymorphism in the exon 4-intron 5 boundary region of the CYP19 gene have been investigated in breast cancer by several studies, with inconsistent results $[1,2]$. For both polymorphisms the variant alleles are considered to be related to an increased biosynthesis of endogenous estrogen. The CYP17 $\mathrm{T} \rightarrow \mathrm{C}$ polymorphism is thought to create an Sp1-type (CCACC) promoter site (although one study did not confirm this [3]) and is associated with an increased serum estrogen level $[4,5]$. After Feigelson and colleagues first published their study [6] showing a higher risk of breast cancer in relation to the CYP17 C allele among nonCaucasian women, many other authors attempted to replicate this in other populations. Although some studies confirmed this initial finding, others did not. All studies reporting an increased risk, including the original study, found the increased risk in one or more certain subgroups of women studied, for example women with advanced disease [6], women aged less than 40 years [7], women aged less than 40 years with family history [8], women aged more than 55 years [9], and women also carrying other genetic polymorphisms [10]. Two studies found that women carrying CYP17 C allele are less likely to use hormone replacement therapy $[5,11]$ and three studies found that the protective effect of later age at menarche is stronger among women who do not carry the $\mathrm{C}$ allele $[5,6,12]$. A recent meta-analysis concluded that the CYP17 $\mathrm{T} \rightarrow \mathrm{C}$ polymorphism is not a significant independent risk factor for breast cancer [2].

The CYP19 gene contains a variable number (range 7-13) of TTTA repeats in the exon 4-intron 5 boundary region, creating polymorphisms that have been examined in five studies [13-17]. Kristensen and colleagues [13] and subsequently others found a roughly twofold to fourfold elevated risk in relation to certain numbers of CYP19 TTTA repeat polymorphisms. Although one small study found a higher risk in relation to the TTTA seven repeats allele, $(\text { TTTA })_{7}$ [14], most studies reporting an association found elevated risks in relation to one of the higher number of TTTA repeat alleles: 10 repeats, (TTTA) ${ }_{12}$ [13]; 12 repeats, $(\text { TTTA })_{10}[15,16]$; or 10 or more repeats, (TTTA) $\geq 10$ [17]. A meta-analysis published in 1999 based on some of the earlier studies found that women carrying the CYP19 (TTTA) 10 allele were at higher risk of breast cancer [1].

All published studies of association between the CYP17 and CYP19 polymorphisms and breast cancer discussed above used a classical case-control design. A recent metaanalysis of $\mathrm{CYP} 17 \mathrm{~T} \rightarrow \mathrm{C}$ polymorphism indicates substantial differences in genotype frequencies in case-control studies conducted in different populations [2], with proportions of carriers ranging from 0.46 in the UK [18] to 0.79 in Japan [19] and proportions of homozygotes ranging from $11 \%$ in Finland [12] to $36 \%$ in Taiwan [10]. Similarly, the allele frequency of the CYP19 (TTTA) 10 allele ranges from $0.5 \%$ [15] to $1.8 \%$ [14]. Given that case-control studies can be susceptible to population stratification bias, it is important to examine these potentially important biologically plausible hypotheses in family-based studies that are free from such bias. In this study we examine the association between the CYP17 promoter $\mathrm{T} \rightarrow \mathrm{C}$ and CYP19 TTTA repeat polymorphisms and female breast cancer by using a family-based design among nuclear families participating in the Metropolitan New York Registry (MNYR), one of the six international centers of the National Cancer Institute's Breast Cancer Family Registry project. Although other polymorphisms in the CYP17 and CYP19 genes have been reported, we focused on these two polymorphisms because they have been studied most extensively both in relation to their potential associations with breast cancer and also in relation to their influence on circulating estrogens.

All published studies focused on the relationship between a woman's own constitutional genotype and her breast cancer risk. A body of recent literature has provided limited data suggesting that a woman's breast cancer risk might be related not only to her own endogenous estrogens during adolescence and adulthood, but also to her prenatal exposure; that is, her exposure in utero to her maternal circulating estrogens [20-25]. In addition to the main association between a woman's own genotype and her breast cancer status, the family-based design of the present study allows us to address this hypothesis indirectly, by examining the association between maternal carrier status of CYP17 or CYP19 gene variants (that is, exposure in utero to an altered level of maternal estrogens) and breast cancer status in daughters.

\section{Methods}

\section{Selection of study participants}

Since 1995 the MNYR has been recruiting families with breast and/or ovarian cancers in clinical and community settings within the metropolitan New York area. Families meeting one or more of the following criteria are invited to participate: a female less than 45 years of age at diagnosis of breast cancer; a female with both breast and ovarian cancer; three or more relatives with breast or ovarian cancer diagnosed at age 45 years or more, or any male with breast cancer. After identification of a proband he/she is invited to participate in the registry and his/her family's eligibility is assessed. If the family is eligible and the proband agrees to participate, after appropriate informed consent, he/she is interviewed either in person or by phone with an epidemiology questionnaire and a family-history 
questionnaire. The proband is also asked to provide permission to contact family members. Blood or buccal samples are also collected and participants are provided with a self-administered dietary questionnaire to be returned by mail. Once family members consent to participate, data and blood or buccal samples from the family members are also collected in a similar manner. For members affected with cancer, tumor tissue samples are collected and reviewed pathologically. Genomic DNA from white blood cells or buccal samples has been collected for participants who donated biological samples. So far, the MNYR has enrolled 1158 families and more than 3900 total participants.

For this study we restricted attention to nuclear families with at least one affected daughter and at least one parent and/or sibling for whom DNA samples were available. Of the 1158 families enrolled in the MNYR so far, 278 families met these eligibility criteria. Subjects can participate in the MNYR with or without completion of the full epidemiology questionnaire and/or blood samples. There were 1123 family members in the 278 eligible nuclear families, of whom 702 completed the full epidemiology questionnaire and provided blood samples. However, accurate data on relevant variables for the statistical method used in this study (see below) for the remaining 421 members were available from the family-history questionnaire completed by the 702 members. There was $99 \%$ concordance in data on age and affected status between women who completed the full epidemiology questionnaire and women who did not.

\section{Laboratory analysis}

We evaluated association between the $\mathrm{T} \rightarrow \mathrm{C}$ single-nucleotide polymorphism in the promoter region of the CYP17 gene and the tetranucleotide (TTTA) repeat polymorphism in the exon 4-intron 5 boundary of the CYP19 gene. A total of 23 subjects could not be genotyped for CYP17, and 26 subjects could not be genotyped for CYP19. Genotype data were available on a total of 679 members (from 277 nuclear families) for CYP17 and 676 members (from 278 nuclear families) for CYP19.

The CYP17 promoter polymorphism was determined with template-directed primer extension and detection by fluorescence polarization in a 96-microwell-based format $[26,27]$. In brief, DNA isolated from blood cells by salting out was used for genotyping subjects. First, the target DNA was amplified by polymerase chain reaction (PCR; using forward primer 5'-TTTAAAAGGCCTCCTTGTGC-3' and reverse primer 5'-TTGGGCCAAAACAAATAAGC-3') to generate products in the range 100-200 base pairs. After amplification by PCR, the primers were digested with shrimp alkaline phosphatase and Escherichia coli exonuclease I. Then single-nucleotide extension was performed in the presence of the appropriate allele-specific ddNTPs differentially fluorescence-labeled with either R110 or tetramethylrhodamine purchased from NEN Life Sciences (Boston, MA). For the single-nucleotide extension reaction both forward and reverse probes were tested to select the optimum (the forward probe 5'-GCCACAGCTCTTCTACTCCAC-3') on the basis of clear signal differences. The incorporation resulted in diminished rotation of the fluor compared with the ddNTP. Finally, the fluorescence polarization was read on a fluorescence polarization microplate reader (Tecan Polarion, Research Triangle Park, NC). The reader generates the genotype data on the basis of the distinct separations (with appropriate cut-offs) of the fluorescent intensity values for different alleles in comparison with internal controls.

The CYP19 TTTA repeats were determined by PCR amplification (using the forward primer 5'-GTCTATGAATGTGCCTTTTT-3' and the reverse primer 5'GTTTGACTCCGTGTGTTTGA-3') followed by analysis on an $A B I 377$ system with GenScan software on the basis of the separations on gel according to the differences in the number of TTTA repeats.

All laboratory assays were performed with laboratory personnel blinded to the subject's disease status or family relationships. In addition to assay-specific quality-control samples, $10 \%$ of samples were reassayed after relabeling to keep laboratory staff blinded to its identity.

\section{Statistical analysis}

We used the Family Genetic Analysis Program (FGAP [28], freely available at http://www.stanford.edu/dept/ HRP/epidemiology/FGAP) to test the null hypothesis of no association between genotype and breast cancer risk in nuclear families. The FGAP computes two test statistics: the nonfounder statistic (NFS), a generalization of the transmission disequilibrium test (TDT) $[29,30]$, which evaluates transmission disequilibrium from parents to offspring, and the founder statistic (FS), which compares the distribution of parental genotypes with that expected under the null hypothesis of no association. The FGAP statistics fully exploit data from families with variable numbers of affected/ unaffected members with variable (known/unknown) patterns of parental genotypes. They are similar to, but can be more powerful than, those available in the software FBAT [31]. (See [32] for a comparison of the methods.)

On the basis of the previous evidence $[6,13,15,17]$, we hypothesized that breast cancer risk is elevated among carriers of the CYP17 $\mathrm{C}$ allele and the CYP19 variant alleles with 10 or more TTTA repeats, namely the (TTTA) 10 , $(\text { TTTA })_{11},(\text { TTTA })_{12}$, and (TTTA $)_{13}$ alleles. The data analysis was focused on two specific components of the study hypotheses: first, whether a woman's carrier status of the hypothesized alleles is associated with her breast cancer 
status, and second, whether a mother's carrier status of the hypothesized alleles is associated with her daughter's breast cancer risk. For testing the first component of a hypothesis, we applied the FS and NFS to assess whether specific genotypes of each of the studied genes are related to breast cancer. Because FS and NFS follow a normal Gaussian distribution under the null hypothesis, the assessment of statistical significance of the association can be done on the basis of the deviation of these statistics from the standard critical values under normal distribution.

For simplicity, we describe these analyses for the CYP17 gene as applied to nuclear families consisting of two parents and at least one daughter. Parents may be untyped and the mother's breast cancer status may be unknown. The test statistics, which are likelihood-based score statistics, are obtained by summing the score contributions from each family. These family-specific scores are obtained in three steps.

In the first step we imputed a probability distribution for the genotypes of each pair of parents, conditional on the observed genotypes of all family members. To do this, we obtained maximum-likelihood estimates of the genotypes TT, TC and CC for each of a pair of parents, given the observed genotypes in the family. These estimates do not require the assumption of Hardy-Weinberg frequencies for parental genotypes. If, for example, both parents' genotypes were known, then the probabilities are degenerate at the observed genotypes. Similarly, if both parents' genotypes were unknown but two offspring had observed CYP17 genotypes TT and CC, then the parental distributions are degenerate at TC because both parents must be heterozygotic.

In the second step we used the inferred parental genotype distribution and the offspring's observed genotypes to test whether heterozygous parents were equally likely to transmit $T$ and $C$ alleles to affected daughters. This evaluation is based on the NFS. Under the null hypothesis of equal transmission of $\mathrm{T}$ and $\mathrm{C}$ alleles from parents to affected daughters, the NFS has an asymptotic standard Gaussian distribution. The NFS generalizes the TDT to families with untyped parents and to families with both affected and unaffected daughters. It can be considerably more powerful than the sibling TDT test [33] when applied to families without unaffected daughters.

In the final step we used the inferred parental genotypes (and the mothers' breast cancer phenotypes) in the FS to compare the parental genotype distribution with the expected distribution under the null hypothesis of no association. This statistic treats the affected and unaffected mothers like cases and controls in a case-control study. However, each parent's contribution is weighted in propor- tion to his/her number of affected and unaffected daughters, so that parents of many affected daughters receive higher weights than do those of few affected daughters.

To test the second component of our hypothesis, namely the association between maternal carrier status and daughter's breast cancer status, we evaluated whether the genotypes of mothers with more affected daughters differ from those of mothers with less affected daughters. Such deviation might be expected if some aspect of a daughter's environment in utero, governed by the mother's genotype, influences the daughter's risk of subsequent breast cancer development. The FS was adapted to evaluate this question by comparing the observed or imputed genotypes of mothers of affected daughters with the genotypes expected in the parental population. It is a weighted sum of differences between each mother's observed (or inferred) $\mathrm{C}$ allele count and the average $\mathrm{C}$ count in the population. In symbols, each family's contribution to this sum is proportional to the quantity $\left(n_{\mathrm{A}}-n_{\mathrm{U}}\right)\left(C_{\mathrm{obs}}-C_{\text {exp }}\right)$, where $n_{\mathrm{A}}$ and $n_{\mathrm{U}}$ are,, respectively, the numbers of affected and unaffected daughters in the family, and $C_{\text {obs }}$ and $C_{\text {exp }}$ are the observed and expected $\mathrm{C}$-allele counts for the mother. Under the null hypothesis of no association between maternal genotype and daughters' breast cancer risks, $C_{\text {obs }}$ has a mean value $C_{\text {exp }}$, so $C_{\text {obs }}-C_{\text {exp }}=0$ in expectation for all families. Thus the FS has expectation zero and the correct type I error rate regardless of the actual numbers of affected and unaffected daughters in each family. Under the alternative hypothesis that maternal $\mathrm{C}$-allele count is associated with daughters' breast cancer risks, one expects that $C_{\text {obs }}-C_{\text {exp }}$ $>0$, and thus families with many affected daughters and few unaffected daughters (that is, $n_{\mathrm{A}}-n_{U} \gg 0$ ) contribute larger values to the FS than those with few affected daughters or those with many unaffected daughters. A statistically significant value of the FS when restricted to the mothers (with an insignificant value when restricted to the fathers) would provide evidence for this association.

When the null hypothesis is rejected, it is useful to estimate a measure of association between genotype and risk, such as the odds ratio, and to evaluate the effects of potential confounding by hormonal factors. To do so, we also performed conditional logistic regression analyses $[34,35]$ on all the available sibships containing at least one affected sibling and at least one unaffected sibling who had provided blood samples and relevant epidemiology data for statistical adjustment (165 sibships for CYP17 and 169 sibships for CYP19).

\section{Results}

Of the 277 nuclear families eligible for CYP17 analyses, 229 were Caucasian, 4 were African American, 41 were Hispanic, and 3 were Asian American. Of the 278 nuclear families eligible for CYP19 analyses, 229 were Caucasian, 
Table 1

Genotype distribution of the study population by gender, family relationship and affected status

\begin{tabular}{|c|c|c|c|c|c|c|c|}
\hline \multirow[b]{2}{*}{ Genotype } & \multicolumn{2}{|c|}{ Mothers } & \multicolumn{2}{|c|}{ Daughters } & \multirow[b]{2}{*}{ Fathers } & \multirow[b]{2}{*}{ Sons } & \multirow[b]{2}{*}{ Total } \\
\hline & Affected & Unaffected & Affected & Unaffected & & & \\
\hline \multicolumn{8}{|l|}{ CYP17 } \\
\hline $\mathrm{CC}$ & 2 & 10 & 47 & 41 & 6 & 11 & 117 \\
\hline СТ & 8 & 32 & 147 & 108 & 30 & 22 & 347 \\
\hline $\mathrm{TT}$ & 2 & 19 & 107 & 61 & 8 & 18 & 215 \\
\hline Unknown & 64 & 140 & 0 & 0 & 233 & 0 & 437 \\
\hline Total & 76 & 201 & 301 & 210 & 277 & 51 & 1116 \\
\hline \multicolumn{8}{|c|}{ CYP19 (no. of TTTA repeats) } \\
\hline $7 / 7$ & 1 & 15 & 88 & 55 & 7 & 21 & 187 \\
\hline $7 / 8$ & 0 & 10 & 34 & 29 & 4 & 5 & 82 \\
\hline $7 / 9$ & 0 & 0 & 1 & 0 & 1 & 0 & 2 \\
\hline $7 / 10$ & 0 & 1 & 5 & 6 & 2 & 0 & 14 \\
\hline $7 / 11$ & 9 & 19 & 106 & 71 & 14 & 19 & 238 \\
\hline $7 / 12$ & 0 & 2 & 9 & 8 & 0 & 0 & 19 \\
\hline $7 / 13$ & 0 & 0 & 0 & 0 & 1 & 0 & 1 \\
\hline $8 / 8$ & 0 & 0 & 4 & 1 & 0 & 0 & 5 \\
\hline $8 / 10$ & 0 & 1 & 3 & 2 & 0 & 0 & 6 \\
\hline $8 / 11$ & 1 & 2 & 24 & 16 & 1 & 3 & 47 \\
\hline $8 / 12$ & 0 & 0 & 1 & 2 & 0 & 0 & 3 \\
\hline $8 / 13$ & 0 & 0 & 0 & 1 & 0 & 0 & 1 \\
\hline $9 / 11$ & 0 & 0 & 0 & 1 & 0 & 0 & 1 \\
\hline $10 / 11$ & 0 & 0 & 2 & 3 & 0 & 2 & 7 \\
\hline $11 / 11$ & 0 & 5 & 19 & 16 & 1 & 1 & 42 \\
\hline $11 / 12$ & 0 & 1 & 4 & 2 & 0 & 0 & 7 \\
\hline $11 / 13$ & 1 & 0 & 2 & 1 & 1 & 0 & 5 \\
\hline $11 / \operatorname{not} 11^{\mathrm{a}}$ & 1 & 3 & 0 & 0 & 5 & 0 & 9 \\
\hline Unknown & 63 & 143 & 0 & 0 & 241 & 0 & 447 \\
\hline Total & 76 & 202 & 302 & 214 & 278 & 51 & 1123 \\
\hline
\end{tabular}

alndicates those whose genotype cannot be inferred for both alleles; the other allele could be 7,8 , or 12 . Two of these nine observations, one an unaffected mother and the other the father in the same nuclear family, will be excluded when the allele with 10 or more repeats is selected as bad allele, because either them could be $11 / 12$.

4 were African American, 42 were Hispanic, and 3 were Asian American. Table 1 shows the distribution of the study subjects according to CYP17 and CYP19 genotypes, by family position and breast cancer status. The numbers in each cell represent the number of specific type of family members in our study population carrying a particular genotype. The number of TTTA repeats in intron 4 of the CYP19 gene ranged between 7 and 13 in our study population, with the (TTTA $)_{7}$ and (TTTA) ${ }_{11}$ alleles being the most frequent (allele frequencies $53.9 \%$ and $28.8 \%$, respec- tively). These frequencies are consistent with those found in Caucasian populations in other studies in the USA [15]. The frequency of the CYP17 variant C allele was $42.8 \%$ in this study population, which is similar to that found in other studies conducted in Caucasians [4].

The distribution of the nuclear families according to mother's and father's carrier status and mother's and daughter's affected status is presented in Table 2. A majority (about $55 \%$ ) of the nuclear families contained one 
Table 2

Distribution of participating nuclear families according to mother's breast cancer status, mother's and father's carrier status of the CYP17 and CYP19 variant alleles, and number of affected and unaffected daughters

\begin{tabular}{|c|c|c|c|c|c|c|c|c|c|c|c|}
\hline \multirow{3}{*}{$\begin{array}{l}\text { Number of daughters } \\
\text { affected/unaffected }\end{array}$} & \multicolumn{6}{|c|}{$\begin{array}{l}\begin{array}{l}\text { Number of nuclear families according to mother's breast cancer status and } \\
\text { genotype }\end{array}\end{array}$} & \multirow{3}{*}{ Total } & \multicolumn{4}{|c|}{$\begin{array}{l}\text { Number of nuclear families according to father's } \\
\text { genotype }\end{array}$} \\
\hline & & Affected & & & Unaffected & & & & & & Total \\
\hline & Carrier & Non-carrier & Unknown & Carrier & Non-carrier & Unknown & & Carrier & Non-carrier & Unknown & \\
\hline \multicolumn{12}{|l|}{ CYP17 } \\
\hline $1 / 0$ & 3 & 0 & 19 & 17 & 12 & 29 & 80 & 12 & 2 & 66 & 80 \\
\hline $1 / 1$ & 5 & 2 & 38 & 16 & 6 & 85 & 152 & 19 & 4 & 129 & 152 \\
\hline $1 / 2$ & 0 & 0 & 2 & 4 & 0 & 14 & 20 & 4 & 2 & 14 & 20 \\
\hline $1 / 3$ & 0 & 0 & 1 & 2 & 0 & 0 & 3 & 0 & 0 & 3 & 3 \\
\hline $2 / 0$ & 2 & 0 & 3 & 1 & 1 & 9 & 16 & 0 & 0 & 16 & 16 \\
\hline $2 / 1$ & 0 & 0 & 0 & 0 & 0 & 2 & 2 & 0 & 0 & 2 & 2 \\
\hline $2+/ 2+$ & 0 & 0 & 1 & 2 & 0 & 1 & 4 & 1 & 0 & 3 & 4 \\
\hline Total & 10 & 2 & 64 & 42 & 19 & 140 & 277 & 36 & 8 & 233 & 277 \\
\hline \multicolumn{12}{|l|}{ CYP19 $>=10$ repeats } \\
\hline $1 / 0$ & 4 & 0 & 17 & 14 & 13 & 29 & 77 & 7 & 4 & 66 & 77 \\
\hline $1 / 1$ & 5 & 1 & 40 & 12 & 9 & 88 & 155 & 10 & 6 & 139 & 155 \\
\hline $1 / 2$ & 1 & 0 & 1 & 5 & 1 & 14 & 22 & 5 & 2 & 15 & 22 \\
\hline $1 / 3$ & 1 & 0 & 0 & 1 & 0 & 0 & 2 & 1 & 0 & 1 & 2 \\
\hline $2 / 0$ & 1 & 0 & 4 & 1 & 1 & 9 & 16 & 1 & 0 & 15 & 16 \\
\hline $2 / 1$ & 0 & 0 & 0 & 0 & 0 & 2 & 2 & 0 & 0 & 2 & 2 \\
\hline $2+/ 2+$ & 0 & 0 & 1 & 0 & 1 & 2 & 4 & 0 & 0 & 4 & 4 \\
\hline Total & 12 & 1 & 63 & 33 & 25 & 144 & 278 & 24 & 12 & 242 & 278 \\
\hline \multicolumn{12}{|l|}{ CYP19 $=11$ repeats } \\
\hline $1 / 0$ & 4 & 0 & 17 & 11 & 16 & 29 & 77 & 6 & 5 & 66 & 77 \\
\hline $1 / 1$ & 5 & 1 & 40 & 11 & 10 & 88 & 155 & 9 & 7 & 139 & 155 \\
\hline $1 / 2$ & 1 & 0 & 1 & 6 & 1 & 13 & 22 & 5 & 3 & 14 & 22 \\
\hline $1 / 3$ & 1 & 0 & 0 & 1 & 0 & 0 & 2 & 1 & 0 & 1 & 2 \\
\hline $2 / 0$ & 1 & 0 & 4 & 1 & 1 & 9 & 16 & 1 & 0 & 15 & 16 \\
\hline $2 / 1$ & 0 & 0 & 0 & 0 & 0 & 2 & 2 & 0 & 0 & 2 & 2 \\
\hline $2+/ 2+$ & 0 & 0 & 1 & 0 & 1 & 2 & 4 & 0 & 0 & 4 & 4 \\
\hline Total & 12 & 1 & 63 & 30 & 29 & 143 & 278 & 22 & 15 & 241 & 278 \\
\hline
\end{tabular}

affected and one unaffected daughter. The majority of the nuclear families had one or more parents who did not have the genotyping information available.

Table 3 presents the FS and NFS for testing the associations between the a priori hypothesized CYP17 and CYP19 variant alleles and breast cancer. Each test statistic has an approximately standard Gaussian distribution under the null hypothesis of no association between genotype and breast cancer risk. A positive value of a NFS reflects excess transmission of the variant allele to affected daughters, and a negative value represents fewer such transmissions than expected under the null. Thus a test statistic that is negative but large in absolute value would suggest that the variant allele is associated with reduced risk. We computed the FS and NFS under recessive, dominant, and additive models. For the dominant models, the number of affected daughters carrying one or more copies of the variant alleles was compared with that expected from the parental genotypes in accordance with Mendelian expecta- 
Association between the CYP19 and CYP17 variant alleles and breast cancer

\begin{tabular}{|c|c|c|c|c|c|c|c|}
\hline \multirow[b]{2}{*}{ Variant allele(s) } & \multirow[b]{2}{*}{$\begin{array}{l}\text { Estimated allele } \\
\text { frequency (\%) }\end{array}$} & \multicolumn{3}{|c|}{ Nonfounder statistic } & \multicolumn{3}{|c|}{ Founder statistic } \\
\hline & & Recessive model & Dominant model & Additive model & Recessive model & Dominant model & Additive model \\
\hline \multicolumn{8}{|l|}{ CYP17 } \\
\hline C & 42.46 & -1.01 & -1.52 & -1.85 & 0.40 & 1.08 & 1.01 \\
\hline$P$ & & 0.16 & 0.06 & 0.03 & 0.34 & 0.14 & 0.16 \\
\hline \multicolumn{8}{|l|}{ CYP19 } \\
\hline$(\mathrm{TTTA})_{\geq 10}$ & 33.71 & -1.24 & 1.26 & 0.32 & -0.32 & 1.66 & 1.13 \\
\hline$P$ & & 0.11 & 0.10 & 0.38 & 0.37 & 0.05 & 0.13 \\
\hline$(\mathrm{TTTA})_{11}$ & 28.78 & -1.09 & 1.83 & 0.97 & -1.50 & 1.96 & 0.89 \\
\hline$P$ & & 0.14 & 0.03 & 0.17 & 0.07 & 0.03 & 0.19 \\
\hline
\end{tabular}

$P$ values are based on one-tailed test statistics. Values that are statistically significant at one-tailed test are displayed in bold type.

tion. Similarly, for the recessive models, the number of affected daughters homozygous for the variant allele was compared with that expected under Mendelian expectation. For the additive models, the total variant allele count in affected daughters was compared with that expected from the parental genotypes in accordance with Mendelian expectation. On the basis of the literature, we hypothesized a priori that CYP19 alleles with 10 or more TTTA repeats would be associated with breast cancer. In addition, we examined the association between the CYP19 genotype and breast cancer by defining the variant allele(s) by treating each of the 10 or more repeat alleles, (TTTA) $)_{10}$, $(\text { TTTA })_{11},(\text { TTTA })_{12}$ and (TTTA) ${ }_{13}$, separately as the variant allele under each of the three models (realizing that this might have increased the chance of our finding of a statistically significant association; see the Discussion section).

As seen in Table 3, the NFS for association between the (TTTA) ${ }_{11}$ allele and breast cancer under the dominant model is 1.83 , which is higher than the critical value (1.65) for a one-tailed test statistic, suggesting that affected daughters were more likely to receive the (TTTA) ${ }_{11}$ allele from their parents (irrespective of their ethnic distribution) than unaffected daughters. Like the NFS, the FS was also statistically significant under the dominant model, supporting an association between the CYP19 (TTTA) ${ }_{11}$ allele and breast cancer among the parents in these families. The results for CYP19 TTTA T10 $_{10}$ alleles did not show a consistent association, because only the FS was statistically significant under the dominant model. None of the other specific CYP19 alleles showed a consistent association with breast cancer on the basis of the NFS and FS (results not shown). Although the FS found an association between the CYP19 (TTTA) ${ }_{13}$ allele and breast cancer, this was not supported by the more robust NFS (results not shown).
Neither the FS nor the NFS suggested any significant association between the CYP17 variant $C$ allele and breast cancer, under any of the models of FGAP analyses (see Table 3).

Table 4 presents the results of conditional logistic regression analysis comparing the CYP17 and CYP19 genotypes between affected and unaffected sisters. These results, adjusted for age (in years), hormone replacement use (ever/never), oral contraceptive use (ever/never), age at menarche (in years) and term pregnancies (yes/no), are similar to the FGAP results although because of the smaller number of available sibships the associations did not achieve statistical significance. As seen in Table 4, carriers of the CYP19 (TTTA) ${ }_{11}$ allele had an increased risk of breast cancer (odds ratio 1.8; 95\% confidence interval 0.9-3.5).

Table 5 presents results relating maternal and paternal carrier statuses for the variants of estrogen-biosynthesis genes CYP17 and CYP19 to breast cancer risk in daughters. Mothers of affected daughters were more likely to carry the CYP19 (TTTA) ${ }_{11}$ allele than expected in the parental population. There were no such associations between the paternal carrier status of (TTTA ${ }_{11}$ and any of the other CYP19 alleles and breast cancer in daughters. For this hypothesis, the findings for analysis involving CYP19 (TTTA) $)_{\geq 10}$ corroborated that for (TTTA) ${ }_{11}$ alleles. Although maternal carrier status of the CYP17 C allele tended to be positively associated with daughter's breast cancer, this association was not specific to the mothers but was also present among the fathers.

\section{Discussion}

Despite a sound biological basis for the role of estrogenbiosynthesis genes in breast cancer, the findings of studies investigating the relationship between these genes and 
Table 4

Conditional logistic regression analysis of discordant sibships for the association between CYP17 and CYP19 genotypes and breast cancer

\begin{tabular}{|c|c|c|c|c|}
\hline Gene (sibling sets/cases/controls) & & Affected $(n)$ & Unaffected $(n)$ & $\begin{array}{l}\text { Adjusted odds ratios for } \\
\text { breast cancer }(95 \% \mathrm{Cl})\end{array}$ \\
\hline \multicolumn{5}{|l|}{ CYP17 (165/171/188) } \\
\hline \multirow[t]{2}{*}{ Dominant model } & $\mathrm{TT}$ & 59 & 56 & 1.00 \\
\hline & $\mathrm{TC} / \mathrm{CC}$ & 112 & 132 & $0.86(0.47-1.59)$ \\
\hline \multirow[t]{2}{*}{ Recessive model } & $\mathrm{TC} / \mathrm{TT}$ & 146 & 154 & 1.00 \\
\hline & $\mathrm{CC}$ & 25 & 34 & $0.61(0.27-1.41)$ \\
\hline \multirow[t]{3}{*}{ General model } & $\mathrm{TT}$ & 59 & 56 & 1.00 \\
\hline & TC & 87 & 98 & $0.86(0.47-1.59)$ \\
\hline & $\mathrm{CC}$ & 25 & 34 & $0.55(0.21-1.42)$ \\
\hline \multicolumn{2}{|l|}{ Additive model (trend per allele) } & & & $0.77(0.49-1.21)$ \\
\hline \multicolumn{5}{|c|}{ CYP19 (no. of TTTA repeats) $(169 / 175 / 193)$} \\
\hline \multirow[t]{2}{*}{ Dominant model } & $(\text { TTTA })_{<10}(\text { TTTA })_{<10}$ & 67 & 78 & 1.00 \\
\hline & $(\mathrm{TTTA})_{\geq 10}(\mathrm{TTTA})_{<10} /(\mathrm{TTTA})_{\geq 10}(\mathrm{TTTA})_{\geq 10}$ & 108 & 115 & $1.24(0.63-2.46)$ \\
\hline \multirow[t]{2}{*}{ Recessive model } & $(\mathrm{TTTA})_{<10}(\mathrm{TTTA})_{<10} /(\mathrm{TTTA})_{\geq 10}(\mathrm{TTTA})_{<10}$ & 159 & 173 & 1.00 \\
\hline & $(\mathrm{TTTA})_{\geq 10}(\mathrm{TTTA})_{\geq 10}$ & 16 & 20 & $0.82(0.30-2.24)$ \\
\hline \multirow[t]{3}{*}{ General model } & $(T T T A)_{<10}(T T T A)_{<10}$ & 67 & 78 & 1.00 \\
\hline & $(T T T A)_{\geq 10}(T T T A)_{<10}$ & 92 & 95 & $1.26(0.64-2.51)$ \\
\hline & $(\mathrm{TTTA})_{\geq 10}(\mathrm{TTTA})_{\geq 10}$ & 16 & 20 & $0.98(0.30-3.18)$ \\
\hline Additive model (trend per allele) & & & & $1.11(0.65-1.89)$ \\
\hline \multirow[t]{2}{*}{ Dominant model } & $(\mathrm{TTTA})_{\text {other }}(\mathrm{TTTA})_{\text {other }}$ & 77 & 95 & 1.00 \\
\hline & $(\text { TTTA })_{11}(\text { TTTA })_{\text {other }} /(\text { TTTA })_{11}(\text { TTTA })_{11}$ & 98 & 98 & $1.77(0.90-3.47)$ \\
\hline \multirow[t]{2}{*}{ Recessive model } & $(\mathrm{TTTA})_{\text {other }}(\mathrm{TTTA})_{\text {other }} /(\mathrm{TTTA})_{11}(\mathrm{TTTA})_{\text {other }}$ & 165 & 179 & 1.00 \\
\hline & $(\mathrm{TTTA})_{11}(\mathrm{TTTA})_{11}$ & 10 & 14 & $0.66(0.19-2.33)$ \\
\hline \multirow[t]{3}{*}{ General model } & $(T T T A)_{\text {other }}(T T T A)_{\text {other }}$ & 77 & 95 & 1.00 \\
\hline & $(\mathrm{TTTA})_{11}(\mathrm{TTTA})_{\text {other }}$ & 88 & 84 & $1.84(0.93-3.63)$ \\
\hline & $(\mathrm{TTTA})_{11}(\mathrm{TTTA})_{11}$ & 10 & 14 & $1.04(0.27-4.08)$ \\
\hline Additive model (trend per allele) & & & & $1.38(0.79-2.40)$ \\
\hline
\end{tabular}

Odds ratios were adjusted for age (in years), hormone replacement use (ever/never), oral contraceptive use (ever/never), age at menarche (in years), full term pregnancies (yes/no). Each sibling set had at least one breast cancer case and one sister control. All the subjects included in the analysis had information for all the covariate variables. $\mathrm{Cl}$, confidence interval.

breast cancer have not been consistent. Employing a casecontrol design, many of these prior studies, especially those examining the CYP17 gene-breast cancer relationships, produced conflicting results. Although in comparison with CYP17 a smaller number of studies investigated the association of breast cancer with CYP19, findings for CYP19 have been more consistent, with most studies showing a positive association between CYP19 alleles with a higher number $(10,12$, or 10 or more) of TTTA repeats and breast cancer [13,15-17].
Using a family-based design we investigated the relationships between the CYP17 and CYP19 gene variants and breast cancer in families participating in the MNYR. Like many of the previous case-control studies, the present study did not find any association between the CYP17 C (variant) allele and breast cancer. However, our findings support an association between certain alleles of the CYP19 intron 4 TTTA repeat polymorphism and breast cancer. On the basis of the previous studies we defined each of the CYP19 alleles with 10, 11, 12, or 13 TTTA repeats as the 'variant' allele and examined each associa- 
Association between parental carrier status of the variant allele(s) and breast cancer risk in daughters

\begin{tabular}{|c|c|c|c|c|}
\hline \multirow[b]{3}{*}{ Variant allele(s) } & \multicolumn{4}{|c|}{ Test statistic } \\
\hline & \multicolumn{2}{|c|}{ Mothers' carrier status and disease risk in daughters } & \multicolumn{2}{|c|}{ Fathers' carrier status and disease risk in daughters } \\
\hline & Additive & Dominant & Additive & Dominant \\
\hline \multicolumn{5}{|l|}{ CYP17 } \\
\hline $\mathrm{C}$ & 1.47 & 1.09 & 1.40 & 1.07 \\
\hline$P$ & 0.07 & 0.14 & 0.08 & 0.14 \\
\hline \multicolumn{5}{|l|}{ CYP19 } \\
\hline$(\mathrm{TTTA})_{\geq 10}$ & 1.73 & 1.65 & 0.95 & 0.45 \\
\hline$P$ & 0.04 & 0.05 & 0.17 & 0.33 \\
\hline$(\text { TTTA })_{11}$ & 1.52 & 1.96 & -0.11 & 0.18 \\
\hline$P$ & 0.06 & 0.03 & 0.46 & 0.43 \\
\hline
\end{tabular}

The test statistic was calculated under the additive model. $P$ values are based on one-tailed test statistics.

tion with breast cancer. Unlike some of the previous casecontrol studies we did not find the CYP19 (TTTA) ${ }_{10}$ or (TTTA) ${ }_{12}$ alleles to be associated with breast cancer. However, we found the CYP19 (TTTA) ${ }_{11}$ allele to be significantly associated with breast cancer in these nuclear families, under a dominant model. Although we also observed a significantly positive association between the CYP19 (TTTA) ${ }_{13}$ allele and breast cancer among the parents in these families, we did not observe excess transmission from parents to affected daughters, suggesting that the association might be due to chance or bias. The evidence of an increased risk in relation to the CYP19 (TTTA) ${ }_{11}$ allele was also observed in the conditional logistic regression analysis adjusting for potential confounding variables among the subset of families containing discordant sibships. However, because of the reduced power of these analyses among only a subset of families [36], results of these discordant sibship analyses did not achieve statistical significance.

In addition to evaluating associations between a woman's breast cancer risk and her own constitutional genotype, we also evaluated whether maternal genotypes are associated with the breast cancer risk in the daughters (independent of the daughter's own genotype). We found that the maternal (but not the paternal) genotypes of the CYP19 (TTTA) 11 allele conferred a non-significantly elevated breast cancer risk to daughters. This effect was also observed when all $(\text { TTTA })_{\geq 10}$ alleles were treated as the variant allele. This association is consistent with evidence from the previous literature on the association between exposure to hormonal factors in utero and breast cancer risk in adulthood [20]. Although the association might be due to chance, if confirmed in subsequent studies it will have important implica- tions in advancing our understanding of the breast cancer etiology.

Some limitations of the present study merit consideration. The major limitation concerns statistical power. The analysis, which is based on 287 nuclear families, might not have had enough power to detect small increases in risk associated with certain of the CYP17 genotypes. For example, we lacked power to evaluate interactions between genotypes for CYP17 and CYP19 and both endogenous and exogenous hormonal characteristics, such as age at menarche, timing and number of pregnancies and the use of exogenous hormones. In addition, although there is evidence for variations in the allele frequencies of the studied polymorphisms across ethnic groups, we lacked statistical power to conduct ethnicity-specific analyses. The evaluation of such analyses will be the subject of a separate future analysis, based on additional numbers of Breast Cancer Family Registry families.

Although the hypotheses examined in this study are not novel, the study design (which is free from population stratification bias) and the analytical approach have not been applied to these hypotheses in previous studies. Several limitations of this study require caution when interpreting the findings. First, the selection of nuclear families participating in this study from the MNYR was not populationbased. Although this might limit the generalizability of the findings it should not affect the validity of the observed associations. Second, although it is possible for variations in the number of nucleotide repeats in hormone-related genes to be associated with cancer risk, such an association is less plausible biologically for the TTTA repeat numbers in the CYP19 gene. This is because the TTTA polymorphism is in the intronic region of the gene and so it 
is less likely that the variant alleles of the gene are directly associated with the functional status of endogenous estrogens in the body. Nevertheless, it is possible that one or more of the CYP19 TTTA alleles, including the (TTTA) ${ }_{11}$ allele, are in linkage disequilibrium with other functionally relevant alleles, as suggested by other studies [16]. Third, the present study compared multiple CYP19 TTTA alleles with breast cancer under different models. Although it is possible that multiple comparisons might have led to the observed associations, the consistency of the associations involving the CYP19 (TTTA) ${ }_{11}$ allele across both parents and transmission to offspring as well as the similarity between the associations with both constitutional and maternal genotypes suggest that these findings might have a biological basis. Further, the fact that the association was observed under specific susceptibility models and was consistent with conditional logistic regression analysis might be suggestive of the specificity of the finding.

\section{Conclusion}

This family-based study found that the CYP19 (TTTA) 11 allele is associated with breast cancer risk among families participating in a breast cancer family registry. The study also suggests that maternal carrier status of the CYP19 (TTTA ${ }_{11}$ allele might be associated with breast cancer in daughters in these families. These associations might have important implications for understanding the etiology and risk prediction of breast cancer. However, because this is the first study to report an association with the CYP19 (TTTA) ${ }_{11}$ allele, and because multiple comparisons have been made, the associations reported in this study should be interpreted with caution and need to be confirmed in future family-based studies.

\section{Competing interests}

The author(s) declare that they have no competing interests.

\section{Acknowledgements}

This research was supported by US Department of Defense grant no. DAMD17-00-1-0213 and National Cancer Institute under RFA no. CA95-003, Breast Cancer Family Registry, through cooperative agreement with Columbia University.

\section{References}

1. Dunning AM, Healey CS, Pharoah PD, Teare MD, Ponder BA, Easton DF: A systematic review of genetic polymorphisms and breast cancer risk. Cancer Epidemiol Biomarkers Prev 1999, 8:843-854.

2. Ye Z, Parry JM: The CYP17 MspA1 polymorphism and breast cancer risk: a meta-analysis. Mutagenesis 2002, 17:119-126.

3. Nedelcheva KV, Haraldsen EK, Anderson KB, Lonning PE, Erikstein B, Karesen R, Gabrielsen OS, Borresen-Dale AL: CYP17 and breast cancer risk: the polymorphism in the 5 ' flanking area of the gene does not influence binding to Sp-1. Cancer Res 1999, 59:2825-2828.

4. Feigelson HS, Shames LS, Pike MC, Coetzee GA, Stanczyk FZ, Henderson BE: Cytochrome P450c17 $\alpha$ gene (CYP17) polymorphism is associated with serum estrogen and progesterone concentrations. Cancer Res 1998, 58:585-587.
5. Haiman CA, Hankinson SE, Spiegelman D, Colditz GA, Willett WC, Speizer FE, Kelsey KT, Hunter DJ: The relationship between a polymorphism in CYP17 with plasma hormone levels and breast cancer. Cancer Res 1999, 59:1015-1020.

6. Feigelson HS, Coetzee GA, Kolonel LN, Ross RK, Henderson BE: A polymorphism in the CYP17 gene increases the risk of breast cancer. Cancer Res 1997, 57:1063-1065.

7. Bergman-Jungestrom M, Gentile M, Lundin AC, Wingren S: Association between CYP17 gene polymorphism and risk of breast cancer in young women. Int J Cancer 1999, 84:350-353.

8. Spurdle AB, Hopper JL, Dite GS, Chen X, Cui J, McCredie MR, Giles GG, Southey MC, Venter DJ, Easton DF, Chenevix-Trench G: CYP17 promoter polymorphism and breast cancer in Australian women under age forty years. J Natl Cancer Inst 2000, 92:1674-1681.

9. Miyoshi Y, Iwao K, Ikeda N, Egawa C, Noguchi S: Genetic polymorphism in CYP17 and breast cancer risk in Japanese women. Eur J Cancer 2000, 36:2375-2379.

10. Huang CS, Chern HD, Chang KJ, Cheng CW, Hsu SM, Shen CY: Breast cancer risk associated with genotype polymorphism of the estrogen-metabolizing genes CYP17, CYP1A1, and COMT: a multigenic study on cancer susceptibility. Cancer Res 1999 , 59:4870-4875.

11. Feigelson HS, McKean-Cowdin R, Pike MC, Coetzee GA, Kolonel LN, Nomura AM, Le Marchand L, Henderson BE: Cytochrome P450c17 $\alpha$ gene (CYP17) polymorphism predicts use of hormone replacement therapy. Cancer Res 1999, 59:3908-3910.

12. Mitrunen K, Jourenkova N, Kataja V, Eskelinen M, Kosma VM, Benhamou S, Vainio H, Uusitupa M, Hirvonen A: Steroid metabolism gene CYP17 polymorphism and the development of breast cancer. Cancer Epidemiol Biomarkers Prev 2000, 9:1343-1348.

13. Kristensen VN, Andersen TI, Lindblom A, Erikstein B, Magnus $P$ Borresen-Dale AL: A rare CYP19 (aromatase) variant may increase the risk of breast cancer. Pharmacogenetics 1998 , 8:43-48.

14. Siegelmann-Danieli N, Buetow KH: Constitutional genetic variation at the human aromatase gene (Cyp19) and breast cancer risk. Br J Cancer 1999, 79:456-463.

15. Haiman CA, Hankinson SE, Spiegelman D, De VI, Colditz GA, Willett WC, Speizer FE, Hunter DJ: A tetranucleotide repeat polymorphism in CYP19 and breast cancer risk. Int J Cancer 2000, 87:204-210.

16. Baxter SW, Choong DY, Eccles DM, Campbell IG: Polymorphic variation in CYP19 and the risk of breast cancer. Carcinogenesis 2001, 22:347-349.

17. Miyoshi Y, Iwao K, lkeda N, Egawa C, Noguchi S: Breast cancer risk associated with polymorphism in CYP19 in Japanese women. Int J Cancer 2000, 89:325-328.

18. Young IE, Kurian KM, Annink C, Kunkler IH, Anderson VA, Cohen $\mathrm{BB}$, Hooper ML, Wyllie AH, Steel CM: A polymorphism in the CYP17 gene is associated with male breast cancer. $\mathrm{Br} J$ Cancer 1999, 81:141-143.

19. Hamajima N, Iwata $H$, Obata $Y$, Matsuo $K$, Mizutani M, Iwase $T$, Miura S, Okuma K, Ohashi K, Tajima K: No association of the 5 promoter region polymorphism of CYP17 with breast cancer risk in Japan. Jpn J Cancer Res 2000, 91:880-885.

20. Sanderson M, Williams MA, Daling JR, Holt VL, Malone KE, Self SG, Moore DE: Maternal factors and breast cancer risk among young women. Paediatr Perinat Epidemiol 1998, 12:397-407.

21. Weiss HA, Potischman NA, Brinton LA, Brogan D, Coates RJ, Gammon MD, Malone KE, Schoenberg JB: Prenatal and perinatal risk factors for breast cancer in young women. Epidemiology 1997, 8:181-187.

22. Ekbom A, Hsieh CC, Lipworth L, Adami HQ, Trichopoulos D: Intrauterine environment and breast cancer risk in women: a population-based study. J Natl Cancer Inst 1997, 89:71-76.

23. Potischman N, Troisi R: In-utero and early life exposures in relation to risk of breast cancer. Cancer Causes Control 1999, 10:561-573.

24. Ekbom A, Trichopoulos D, Adami HO, Hsieh CC, Lan SJ: Evidence of prenatal influences on breast cancer risk. Lancet 1992, 340:1015-1018.

25. Sanderson M, Williams MA, Malone KE, Stanford JL, Emanuel I, White E, Daling JR: Perinatal factors and risk of breast cancer. Epidemiology 1996, 7:34-37.

26. Kwok PY: SNP genotyping with fluorescence polarization detection. Hum Mutat 2002, 19:315-323. 
27. Hsu TM, Chen X, Duan S, Miller RD, Kwok PY: Universal SNP genotyping assay with fluorescence polarization detection. Biotechniques 2001, 31:560-568.

28. Whittemore AS, Tu IP: Detection of disease genes by use of family data. I. Likelihood-based theory. Am J Hum Genet 2000, 66:1328-1340.

29. Spielman RS, McGinnis RE, Ewens WJ: Transmission test for linkage disequilibrium: the insulin gene region and insulindependent diabetes mellitus (IDDM). Am J Hum Genet 1993, 52:506-516.

30. Schaid DJ: General score tests for associations of genetic markers with disease using cases and their parents. Genet Epidemiol 1996, 13:423-449.

31. Lange C, DeMeo DL, Laird NM: Power and design considerations for a general class of family-based association tests: quantitative traits. Am J Hum Genet 2002, 71:1330-1341.

32. Whittemore AS, Halpern J: Genetic association tests for family data with missing parental genotypes: a comparison. Genet Epidemiol 2003, 25:80-91.

33. Spielman RS, Ewens WJ: The TDT and other family-based tests for linkage disequilibrium and association. Am J Hum Genet 1996, 59:983-989.

34. Hosmer D, Lemeshow S: Logistic regression for matched casecontrol studies. In Applied Logistic Regression New York: John Wiley \& Sons Inc; 1989:187-213.

35. Breslow NE, Day NE: Statistical Methods in Cancer Research. The Analysis of Case-control Studies. IARC Sci. Publ., No. 32 Volume I. New York: Oxford University Press; 1993.

36. Whittemore AS, Halpern J, Ahsan $\mathrm{H}$ : Covariate adjustment in family-based association studies. Genet Epidemiol in press. 